\title{
Prevalencia de Síntomas Asociados a Trastornos Musculoesqueléticos en Estudiantes de Odontología
}

\author{
Prevalence of Symptoms Associated to Musculoskeletal Disorders in Dental Students
}

\author{
Pamela Acevedo Avila*; Verónica Soto Subiabre*; Cristina Segura Solano ${ }^{* \star}$ \& Cristina Sotomayor Castillo***
}

ACEVEDO, A. P.; SOTO, S. V.; SEGURA, S. C. \& SOTOMAYOR, C. C. Prevalencia de síntomas asociados a trastornos musculoesqueléticos en estudiantes de odontología.Int. J. Odontostomat., 7(1):11-16, 2013.

RESUMEN: Existe una alta prevalencia de dolor musculoesqueletal en odontólogos y estudiantes de odontología alrededor del mundo. El objetivo de esta investigación será determinar la prevalencia de síntomas asociados a trastornos musculoesqueléticos en estudiantes de odontología de la Universidad Austral de Chile. Un cuestionario de auto respuesta fue aplicado a 206 estudiantes entre segundo y quinto año al finalizar el semestre académico. El cuestionario incluyó preguntas de antecedentes generales y de presencia de síntomas según tipo, localización, intensidad, frecuencia y momento del día en que aparecen. El $83 \%$ de los estudiantes reportaron molestias musculoesqueléticas encontrando en general una mayor prevalencia en mujeres. En hombres la zona más reportada fue la espalda media, y en mujeres la zona del cuello y hombro. La mayor intensidad se concentró en mujeres con nivel de dolor 4 y en hombres con nivel 3 (según escala EVA). El momento del día con más molestias fue al terminar el trabajo clínico. Según el año de la carrera que cursan, la mayor prevalencia se observó en cuarto año y el síntoma más frecuente fue el cansancio y la fatiga. El incremento en la carga académica y clínica estaría relacionada con una alta prevalencia de trastornos musculoesqueléticos en estudiantes de odontología.

PALABRAS CLAVE: trastornos musculoesqueléticos, estudiantes de odontología, prevalencia.

\section{INTRODUCCIÓN}

La Organización Mundial de la Salud (OMS, 2004) define el trastorno musculoesquelético (TME) como "los problemas de salud del aparato locomotor, es decir, músculos, tendones, esqueleto óseo, cartílagos, ligamentos y nervios, abarcando desde molestias leves y pasajeras hasta lesiones irreversibles y discapacitantes". La salud musculoesqueletal de los dentistas ha sido objeto de numerosos estudios alrededor del mundo (Al Wassan et al., 2001; Alexopoulos et al., 2004; Bugarín-González et al., 2005; Hayes et al., 2009a; Kierklo et al., 2011; Alexandre et al., 2011; Harutunian et al., 2011), enfocándose principalmente en el dolor corporal; sin embargo, poco se ha investigado acerca de la prevalencia de dolor musculoesqueletal en estudiantes de odontología, momento que pudiera marcar la génesis de los TME.
Cuando el cuerpo humano es sometido a posturas estáticas y repetitivas, durante prolongado tiempo, se pueden iniciar una serie de eventos que pueden derivar en dolor, injuria o algún desorden musculoesqueletal (Valachi \& Valachi, 2003a, 2003b). Ya en el año 1946 un estudio realizado por Biller, encontró que el $65 \%$ de los dentistas experimentaba dolor de espalda (Valachi \& Valachi, 2003a, 2003b). En la revisión sistemática realizada por Hayes et al. (2009a), se mostró una prevalencia de entre un 64 a un $93 \%$, en desórdenes musculoesqueléticos en dentistas de diferentes lugares del mundo, recalcando que estos problemas han demostrado contribuir considerablemente, a reducir la productividad laboral y acortar la vida media de la profesión. Rising et al. (2005), reportaron una prevalencia de un $56-71 \%$ de dolor corporal en estudiantes de odontología, distin-

\footnotetext{
* Escuela de Odontología, Universidad Austral de Chile, Valdivia, Chile.

* Instituto de Odontoestomatología, Facultad de Medicina, Universidad Austral de Chile, Chile.

${ }^{* * *}$ Instituto de Salud Pública, Facultad de Medicina, Universidad Austral de Chile, Chile.
} 
guiendo las zonas más afectadas. Diaz-Caballero et al. (2010), reportó una prevalencia de dolor muscular de un $80 \%$ en estudiantes de odontología, donde también evaluó los factores ergonómicos que causan el dolor muscular.

Debido a estas altas prevalencias de dolor corporal reportadas en estudiantes en diferentes países, y a la falta de conocimiento de estos datos en estudiantes chilenos, el objetivo de este estudio es determinar la prevalencia de síntomas asociados a trastornos musculoesqueléticos en estudiantes de odontología de la Universidad Austral de Chile. Estos datos serán de gran utilidad para aplicar medidas preventivas y disminuir en los profesionales las enfermedades que afectan al aparato locomotor, mejorando con ello la calidad de vida y evitando el ausentismo laboral.

\section{MATERIAL Y MÉTODO}

Se realizó un estudio descriptivo de corte transversal. La población de estudio fue el total de los estudiantes de odontología $(n=282)$ que se encontraban desarrollando actividades clínicas de atención de pacientes y actividades de laboratorio de simulación clínica en las dependencias odontológicas de la Universidad Austral de Chile. Correspondió a estudiantes desde segundo a quinto año, excluyendo a los alumnos de primer año, por no realizar dichas actividades, y a los de sexto año, por encontrarse realizando internado asistencial o seminario de titulación fuera de las dependencias de la clínica. Este estudio fue realizado de manera censal. Las variables estudiadas fueron:

Antecedentes generales: sexo (femenino/masculino); año académico $\left(2^{\circ} / 3^{\circ} / 4^{\circ} / 5^{\circ}\right)$; Antecedentes de sintomatología: tipo de síntoma (dolor/temblores/cansancio o fatiga/parestesia/ausencia); zona corporal afectada por el síntoma (zona lateral del cuello y hombro/zona posterior del cuello y superior de la espalda/ zona media de la espalda/zona baja o lumbar de la espalda/codo/antebrazo/muñecas, manos, dedos); intensidad de la molestia (1-10 EVA); frecuencia de aparición dentro del semestre (rara vez/a menudo/siempre), y momento del día de más sintomatología (al despertar/mientras trabajaba/al terminar el trabajo clínico/al llegar a casa).

Se confeccionó un cuestionario a partir de otro previamente utilizado (Rising et al.), adaptándolo a las necesidades de la investigación y validado sobre un grupo de 13 estudiantes correspondientes a aquellos que estaban cursando sexto año y que habían sido excluidos del estudio. El cuestionario resultó de 10 preguntas, fue anónimo y autorrespondido por los estudiantes de manera presencial. La encuesta se aplicó durante el mes de julio del año 2010, y a cada curso por separado.

Para evitar sesgos por tasa de respuesta y representatividad se utilizó la técnica censal aplicando la encuesta en horarios claves de asistencia obligatoria.

Los datos fueron recolectados y almacenados en planillas computacionales y analizados estadísticamente mediante el software SPSS 18.

\section{RESULTADOS}

El total de estudiantes correspondió a 282, de los cuales 206 respondieron (tasa de respuesta de $73 \%$ ), debido a que no se encontraban presentes el día de la encuesta. El 83\% $(n=171)$ de los estudiantes encuestados reportó algún síntoma asociado a trastornos musculoesqueléticos. Se evaluó a un total de 89 hombres de los cuales el $75 \%$ reportó síntomas de TME. De las 117 mujeres evaluadas, el 89\% registró síntomas de TME. La distribución de hombres y mujeres con sintomatología se observa en la Figura 1, en relación a los distintos niveles académicos. Las zonas del cuerpo más afectadas fueron "espalda media" en los hombres, y "cuello y hombro" en las mujeres. Las tres zonas más reportadas en hombres y mujeres se muestran en la Figura 2.

En mujeres, la intensidad de las molestias se concentró en el nivel 4 en la escala EVA, y en hombres en el nivel 3, que corresponden a niveles medios de molestia. Se reportaron cuatro casos, dos hombres y dos mujeres, con intensidades máximas de 9 y 10 , que se corresponden con una incapacidad para realizar las actividades.

Al inicio del semestre se observó una menor frecuencia de sintomatología expresada en la respuesta "rara vez", lo cual fue aumentando hacia el final del semestre, representado por la frecuencia "a menudo". También se observa que la frecuencia siempre aumentó desde inicio a fin de semestre en un 13\% (Tabla I).

El momento del día en que más se reportaron 


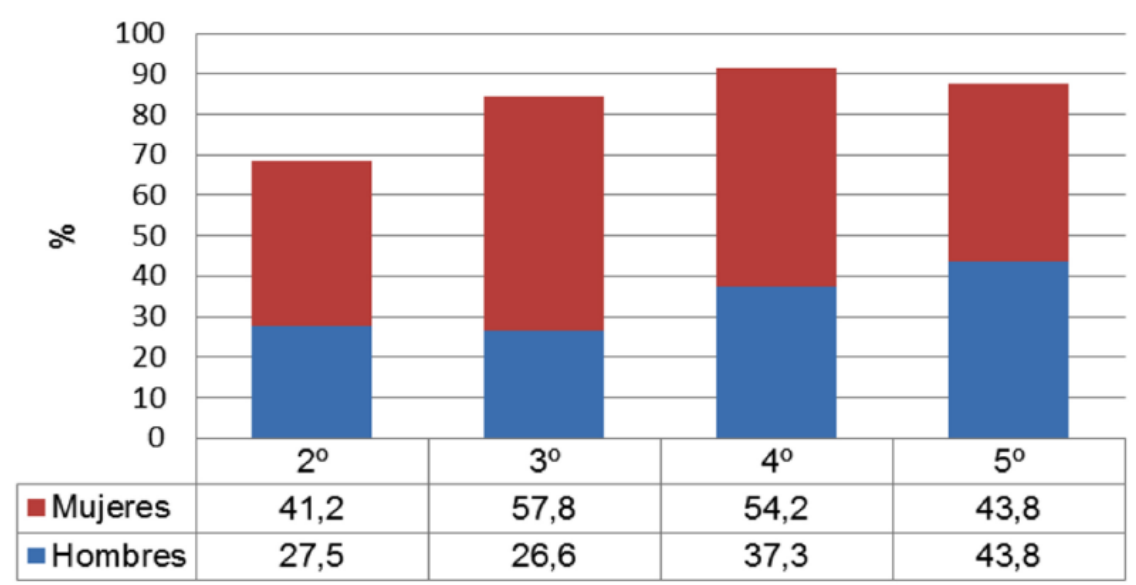

Fig. 1. Prevalencia de alumnos que reportaron síntomas asociados a TME, según año académico y sexo.
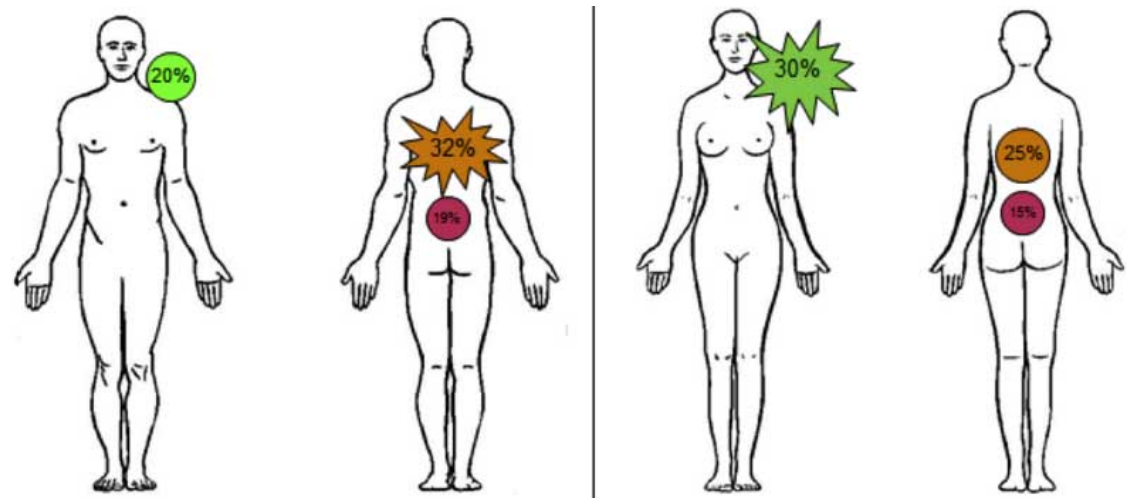

Fig. 2. Zonas del cuerpo con mayor reporte de síntomas según sexo.

Tabla I. Distribución de la frecuencia con que se presentan los síntomas en distintos períodos del semestre.

\begin{tabular}{lccc} 
& $\begin{array}{c}\text { Inicio de } \\
\text { Semestre }(\boldsymbol{\%})\end{array}$ & $\begin{array}{c}\text { Último mes } \\
(\boldsymbol{\%})\end{array}$ & $\begin{array}{c}\text { Últimas 2 } \\
\text { Semanas (\%) }\end{array}$ \\
\hline Rara vez & 66 & 28 & 28 \\
A menudo & 32 & 62 & 57 \\
Siempre & 2 & 10 & 15 \\
\hline
\end{tabular}

Tabla II. Prevalencias de síntomas asociados a TME en estudiantes de odontología reportadas por estudios de diferentes países.

\begin{tabular}{llc}
\hline Est udio & País & Prevalencia \\
\hline Díaz et al. (2010) & Colombia & $80 \%$ \\
De Carvalho et al. (2009) & Brasil & $76 \%$ \\
Hayes et al. (2009b) & Australia & $64 \%$ \\
Thorton et al. (2008) & USA & $61 \%$ \\
Rising et al. (2005) & USA & $46-71 \%$ \\
Tezel et al. $(2005)$ & Turquía & $86 \%$ \\
Acevedo et al. $(2010)$ & Chile & $83 \%$ \\
\hline
\end{tabular}

los síntomas fue "al terminar el trabajo clínico" con un 42,7\%, seguido por "mientras trabajaba" con un $35,6 \%$ y "al llegar a casa" con un $21,6 \%$. No hubo respuesta para la alternativa "al despertar".

La presencia de síntomas fue aumentando junto con los años académicos (Fig. 1), reportándose la mayor prevalencia en los estudiantes de cuarto año (91,5\%), seguido de una leve disminución en quinto $(87,6 \%)$.

Entre todos de estudiantes con molestias, el síntoma más frecuente fue el cansancio o fatiga (83\%), seguido por dolor (44\%), temblores $(9 \%)$ y parestesia $(7 \%)$.

\section{DISCUSIÓN}

Los resultados obtenidos en la Universidad Austral de Chile, en Valdivia, coinciden con los resultados publicados por otros autores, alcanzando una de las prevalencias más altas (Tabla II).

Los síntomas se van incrementando a medida que avanzan los años académicos, presentándose un peak en cuarto año, lo cual coincide con el inicio de atención clínica de pacientes. El inicio de la atención con pacientes reales y el aumento de horas de trabajo clínico, pueden incidir en el incremento de sintomatología en el cuarto año académico. Rising et al., encontraron este mismo aumento de dolor a medida que avanzan los años académicos fluctuando desde un 46 a $71 \%$, lo que se condice también con el inicio de la actividad clínica, tal como en nuestros resultados. Esta disminución podría ser el resultado de una 
adaptación de los estudiantes a las posiciones de trabajo, al mayor dominio de los procedimientos o simplemente por tener menor cantidad de horas de trabajo clínico. En el estudio de Abou-Atme et al. (2007), se realizó un seguimiento a 33 estudiantes de odontología desde primero a quinto año, donde se observó un aumento de los síntomas con un peak en tercer año y una disminución en los posteriores, siguiendo la misma tendencia de los demás estudios, incluido el nuestro. El dolor en la zona de espalda alta aumentó desde primero a tercer año y luego disminuyó significativamente desde tercero a cuarto año $(p=0,043)$; el dolor de espalda baja aumentó de primer a tercer año $(p=0,004)$ y disminuyó de tercer a cuarto año $(p=0,042)$. El estudio es novedoso por el seguimiento realizado, sin embargo, la muestra utilizada fue poco representativa; no vieron diferencias entre hombres y mujeres.

En la mayoría de los estudios no se encuentran diferencias significativas en cuanto al sexo, al igual que nuestros resultados, excepto el de de Carvalho et al. (2009), que reportó una prevalencia significativamente mayor en mujeres $(74,2 \%)$ que hombres $(52,3 \%)$, observándose esta misma tendencia en nuestros resultados.

Las zonas del cuerpo con mayor reporte de molestias en nuestra investigación resultaron también similares a todos los estudios de prevalencia citados (Rising et al.; Tezel et al., 2005; Thorton et al., 2008; Melis et al., 2008; de Carvalho et al.; Hayes et al., 2009b; Diaz-Caballero et al.). Rising et al., reportó significativamente mayor dolor en espalda media en los hombres $(p=0,015)$ y mayor dolor en cuello y hombro en las mujeres $(p=0,004)$, mismos reportes que presentó nuestro estudio. Diaz-Caballero et al., reportaron las zonas más afectadas (15\% para ambos) en cuello-hombro y zona lumbar, sin distinguir entre hombres y mujeres, y Thorton et al., (2008) obtuvo en total mayor prevalencia de dolor en cuello (48\%), espalda $(44 \%)$ y hombro $(31 \%)$, haciendo diferencia por sexo pero no encontrando diferencia significativa. En el estudio de Melis et al., se utilizó un grupo control con estudiantes de psicología y se observó una tendencia mayor de síntomas en todas las zonas corporales evaluadas en los estudiantes de odontología, siendo significativamente mayor sólo el dolor en la zona lumbar $(p<0,05)$, estudio que resulta ser interesante por tener un grupo control de otra profesión.

Rising et al., encontró un valor promedio de dolor en los estudiantes en un rango entre 2,9 y 4,8 (EVA 0-
10), muy similar a nuestros resultados que se concentraron en el nivel 3 en hombres y 4 en mujeres. de Carvalho et al., igualmente obtuvo una intensidad moderada en mujeres y baja en hombres, sin embargo no especifican el método de medición.

La percepción de los síntomas en nuestro estudio va aumentando a medida que avanza el semestre, lo que coincide con el mayor número de evaluaciones teóricas y prácticas, sugiriendo el estrés académico como un factor importante. El momento de mayor molestia fue reportado "durante el trabajo clínico", más que en otro momento del día, tal como reporta De Carvalho et al., esto se puede deber a que los estudiantes trabajen constantemente bajo supervisión y contra el tiempo, sumado a su poca experiencia, lo que aumenta el estrés de trabajo. Thorton et al., (2004) menciona en su estudio que factores estresantes físicos y psicológicos están presentes en el entorno de trabajo dental y que existe una real relación entre el estrés y problemas de salud. Los autores entregan recomendaciones para aplicar un programa de trabajo ergonómico en las escuelas dentales donde los componentes principales a trabajar son: detectar posturas estáticas, movimientos repetitivos, exceso de fuerza; proveer soporte administrativo; realizar cuestionarios para evaluar el riesgo asociado a las tareas especificas de la profesión dental; que la facultad provea un entrenamiento adecuado de los docentes; y desarrollar métodos para reducir o eliminar los factores de riesgo ergonómicos.

En general, se observa una mayor tendencia de presentar síntomas en mujeres que en hombres, lo cual podría ser estudiado desde un punto de vista antropométrico.

Al realizar este estudio no se obtuvo respuesta del total de los estudiantes de odontología de la universidad, sin embargo, sí se trabajó con una tasa de respuesta representativa. Por el diseño metodológico de este estudio, no se pueden establecer relaciones causa-efecto entre las variables, por lo que se recomienda realizar estudios que permitan identificar factores de riesgo que permitan prevenir e intervenir. Sería interesante evaluar otras escuelas de odontología y realizar comparaciones entre ellas, en cuanto a la prevalencia y posibles factores de riesgo, donde sugerimos incluir preguntas relacionadas con la automedicación, especialmente de analgésicos no esteroidales, ya que esto podría estar enmascarando mayores tasas de prevalencia. 


\section{CONCLUSIÓN}

Fue encontrada una alta prevalencia de síntomas en estudiantes que pueden asociarse a futuros desórdenes musculoesqueléticos en los estudiantes de odontología, que podría tener relación con el incremento del trabajo clínico.

La evidencia de distintas partes del mundo, incluyendo la nuestra, muestra resultados similares, lo cual nos da una idea general de la situación que viven los estudiantes de esta profesión. Nos entrega valiosa información para que las escuelas de odontología adopten programas de prevención e intervención, que puedan contribuir a disminuir los trastornos musculoesqueléticos y a mejorar la calidad de vida de los odontólogos.
Es necesario conocer los factores de riesgo implicados en la aparición de síntomas asociados a desordenes musculoesqueletales para poder intervenir en la formación académica de los profesionales y de esta manera reducir la prevalencia, tanto en estudiantes como en odontólogos.

\section{AGRADECIMIENTOS}

Esta investigación está basada en una tesis realizada como requerimiento para la obtención del grado académico de Licenciado en Odontología de la Universidad Austral de Chile, junio, 2012.

ACEVEDO, A. P.; SOTO, S. V.; SEGURA, S. C. \& SOTOMAYOR, C. C. Prevalence of symptoms associated to musculoskeletal disorders in dental students. Int. J. Odontostomat., 7(1):11-16, 2013.

ABSTRACT: There is a high prevalence of musculoskeletal pain in dentists and dental students around the world. The aim of the study is to determine the prevalence of musculoskeletal disorder symptoms in dental students of the Universidad Austral de Chile. A self responded questionnaire was applied to 206 students between second and fifth year, at the end of the academic semester. The questionnaire included questions of general background and symptoms presence depending on type, location, intensity, frequency and time of the day in which they appear. An $83 \%$ of the students reported musculoskeletal discomfort, finding a higher prevalence among women. According to the school year, the highest prevalence was observed in fourth year and the most frequent symptom was tiredness and fatigue. In men, the most reported area was mid back, and in women neck and shoulder. The highest intensity was focused on women with pain level 4 and in men with level 3 (depending on the scale (VAS). The moment of the day with higher discomfort was at the end of the clinical work. A high prevalence of musculoskeletal disorders was found, probably related to the progressive increase of clinical work.

KEY WORDS: musculoskeletal disease, dental student, prevalence.

\section{REFERENCIAS BIBLIOGRÁFICAS}

Abou-Atme, Y. S.; Melis, M.; Zawawi, K. H. \& Cottogno, L. Five-year follow-up of temporomandibular disorders and other musculoskeletal symptoms in dental students. Minerva Stomatol., 56(11-12):603-9, 2007.

Alexandre, P. C.; da Silva, I. C.; de Souza, L. M.; de Magalhães, V.; Palácios, M. \& Meyer, A. Musculoskeletal disorders among Brazilian dentists. Arch. Environ. Occup. Health, 66(4):231-5, 2011.

Alexopoulos, E. C.; Stathi I. C. \& Charizani, F. Prevalence of musculoskeletal disorders in dentist. BMC Musculoskelet. Disord., 5:16, 2004.
Al Wassan, K. A.; Almas, K.; Al Sheltri, S. E. \& Al-Qahtani, M. Q. Back \& neck problems among dentist and dental auxiliaries. J. Contemp. Dent. Pract., 2(3):17-30, 2001.

Bugarín-González, R.; Galeno-Feal, P.; García-García, A. \& Rivas-Lombardero, P. Los trastornos musculoesqueléticos en los odontoestomatólogos. Rev. Ilustre Cons. Gen. Col. Odontol. Estomatol. Esp., 10(56):561-6, 2005.

de Carvalho, M. V.; Soriano, E. P.; de Franças Caldas, A. Jr.; Campello, R. I.; de Miranda, H. F. \& Cavalcanti, F. I. Workrelated musculoskeletal disorders among Brazilian dental students. J. Dent. Educ., 73(5):624-30, 2009. 
Diaz-Caballero, A. J.; Gómez-Cárdenas, I. P. \& Díaz-Cárdenas, S. Ergonomics factors that cause the presence of pain muscle in students of dentistry. Med. Oral Patol. Oral Cir. Bucal,15(6):e906-11, 2010.

Harutunian, K.; Gargallo-Albiol, J.; Figueiredo, R. \& GayEscoda, C. Ergonomics and musculoskeletal pain among postgraduate students and faculty members of the School of Dentistry of the University of Barcelona (Spain). A cross-sectional study. Med. Oral Patol. Oral Cir. Bucal, 16(3):e425-9, 2011.

Hayes, M. J.; Cockrell, D. \& Smith, D. R. A systematic review of musculoskeletal disorders among dental professionals. Int. J. Dent. Hyg., 7(3):159-65, $2009 a$.

Hayes, M. J.; Smith, D. R. \& Cockrell, D. Prevalence and correlated of musculoskeletal disorders among Australian dental hygiene students. Int. J. Dent. Hyg., 7(3):176-81, $2009 b$.

Kierklo, A.; Kobus, A.; Jaworska, M. \& Botulinski, B. Workrelated musculoskeletal disorders among dentists - a questionnaire survey. Ann. Agric. Environ. Med., 18(1):7984, 2011.

Melis, M.; Abou-Atme, Y.; Cottogno, L. \& Pittau, R. Upper body musculoskeletal symptoms in Sardinian dental students. J. Can. Dent. Assoc., 70(5):306-10, 2004.

Rising, D. W.; Bennett, B. C.; Hursh, K. \& Plesh, O. Reports of body pain in a dental student population. J. Am. Dent. Assoc., 136(1):81-6, 2005.

OMS. Serie de protección de la salud de los trabajadores N5. Prevención de trastornos musculoesqueléticos en el lugar de trabajo. Organización Mundial de la Salud 2004. Disponible en: http://www.who.int/ occupational_health/publications/en/pwh5sp.pdf.

Tezel, A.; Kavrut, F.; Tezel, A.; Kara, C.; Demir, T. \& Kavrut, R. Musculoskeletal disorders in left-and-right-handed Turkish dental students. Int. J. Neurosci., 115(2):255-66, 2005.

Thorton, L. J.; Barr, A. E.; Stuart-Buttle, C.; Gaughan, J. P.; Wilson, E. R.; Jackson, A. D.; et al. Perceived musculoskeletal symptoms among dental students in the clinic work environment. Ergonomics, 51(4):573-86, 2008.

Thorton, L. J.; Stuart-Buttle, C.; Wyszynski, T. C. \& Wilson, E. R. Physical and psychosocial stress exposures in US dental schools: the need for expanded ergonomics training. Appl. Ergon., 35(2):153-7, 2004.

Valachi, B. \& Valachi, K. Mechanisms leading to musculoskeletal disorders in dentistry (First in a two-part series). J. Am. Dent. Assoc., 134:1344-50, 2003a.
Valachi, B. \& Valachi, K. Preventing musculoskeletal disorders in clinical dentistry (Second in a two-part series). J. Am. Dent. Assoc., 134:1604-12, 2003b.

Dirección para Correspondencia:

Verónica Soto Subiabre

Escuela de Odontología

Universidad Austral de Chile

Valdivia

CHILE

Email: veronicasoto.y@gmail.com

Recibido : 03-08-2012

Aceptado: 19-11-2012 